\title{
Ziziphus Treatment has a Protective Effect Against Experimentally Induced Osteoporosis in Female Rats
}

\author{
Omar Abdulrhman Alfaroq ${ }^{*}$, Abdulaziz Radhi S ALjohni², Mohamed \\ Hassan Badawood ${ }^{3}$, Sherif Mohamed Hassan ${ }^{4}$, Gamal Said Abdul Aziz \\ and Azeza Abdulrhman Alfaroqie ${ }^{5}$ \\ ${ }^{1}$ Department of Laboratory at King Fahad Hospital, Medina, Saudi Arabia \\ ${ }^{2}$ Department of Laboratory at King Fahad Hospital, Ph.D. Microbiology, Medina, \\ Saudi Arabia \\ ${ }^{3}$ Professor and President of Anatomy Department, King Abdulaziz Student, \\ University, Jeddah, Saudi Arabia \\ ${ }^{4}$ Associate Professor of Anatomy, King Abdulaziz Student, University, Jeddah, Saudi \\ Arabia \\ ${ }^{5}$ Department of Laboratory at King Fahad Hospital, Medina, Saudi Arabia \\ *Corresponding Author: Omar Abdulrahman Alfaroq, Department of Laboratory at \\ King Fahad Hospital, Saudi Arabia.
}

Received: September 16, 2021

Published: November 20, 2021

(C) All rights are reserved by 0 mar

Abdulrhman Alfaroq., et al.

\begin{abstract}
Many investigations have shown that women's transition to menopause might be correlated with greater bone loss. In the medical community, this process is referred to as osteopenia. To find out if the ZSC leaf extract might prevent osteopenia, we looked at the effect of $100 \mathrm{mg} / \mathrm{kg}$ b.wt on the osteopenia of mice. Based on the findings, there was a large rise in insulin, IGF-1, and body weight gain, as well as a significant fall in insulin, IGF-1, and body weight gain. The levels of parathyroid hormone (PTH) and tartrate-resistant acid phosphatase (TRAP) in the blood rose, but the levels of calcitonin (CT), procollagen type 1 (PC1), and osteocalcin (OC) in the blood dropped. A decrease in bone alkaline phosphatase (BALP), bone mineral density (BMD), and calcium and phosphorus levels in both blood and bone are associated with this. It was discovered that the bone of aging rats had increased levels of oxidative stress markers [xanthine oxidase (XOD), hydrogen peroxide $\left(\mathrm{H}_{2} \mathrm{O}_{2}\right)$, nitric oxide (NO), and malondialdehyde (MDA)] as well as decreased levels of antioxidants [glutathione (GSH), superoxide dismutase (SOD), catalase (CAT), and total antioxidant capacity] (TAC). ZSC leaf extract has been found to aid in the reduction of body weight loss and the prevention of all bone alterations. As a result, ZSC leaves may be authorized as a natural medicinal agent with a range of advantages for the treatment of menopause and bone loss, among other conditions.
\end{abstract}

Keywords: King Abdulaziz; University; Jeddah; Ziziphus; Osteoporosis; ZSC Could be Approved as a Natural Therapeutic; Female Rat

\section{Introduction}

Reduced bone mass and microarchitectural degeneration of bone tissue are the symptoms of this skeletal disease, which results in increased bone fragility and fracture susceptibility as a result of the condition's manifestations [1]. Osteoporosis is characterized by increased bone resorption in comparison to bone production, decreased bone mineral density, and decreased trabecular bone volume, to name a few features $[1,2]$.
Osteoporosis can be regarded as serious public health concern since it causes more than 1.3 million fractures each year and incurs financial expenditures of over ten billion dollars in the United States alone each year [2]. Osteoporosis is expected to become an even more serious problem in the future, as the world's population continues to age at an increasing rate. Furthermore, the number of osteoporotic fractures, for example, is growing at a faster rate than can be explained only by demographic changes in some 
countries, such as Finland [3]. Because there are currently no efficient, safe techniques of rebuilding high-quality bone to the osteoporotic skeleton, the prevention of osteoporosis is of paramount importance. Optimization of peak bone mass in early adulthood, as well as prevention of bone loss throughout menopause and as we age, are the cornerstones of osteoporosis preventive strategies. Peak bone mass is influenced by genetic, dietary, and lifestyle variables, and these parameters can be utilized to target preventative measures more effectively. It has been highlighted that a sufficient calcium intake, excellent overall nutrition, and sufficient physical exercise should be followed. When it comes to current therapeutic methods for the prevention of osteoporotic bone loss, the therapies that employ estrogen (+progesterone), bisphosphonates, and calcitonin are the most commonly used by patients. Some recent research has discovered that orally given Ziziphus contains some intriguing characteristics that may be beneficial in the prevention of osteoporosis. A study in rats found that dietary Ziziphus feeding increased calcium and phosphorus levels in the rat's bones. enhance bone calcium replenishment throughout rehabilitation following a calcium deficit in the diet as well as to guard against the loss of bone mineral content caused by ovariectomy in the context of experimental osteoporosis [4-6].

\section{Osteoporosis}

Reduced bone mass, microarchitectural breakdown of bone tissue, increased bone fragility, and an increased risk of fracture are all characteristics of osteoporosis [6]. Furthermore, the importance of factors relating to poor bone quality has been underlined. Osteoporosis is a leading source of morbidity and medical expenditure around the world. It is also important to include negative illness consequences such as pain, sadness, low self-esteem, and loss of independence [6].

Osteoporosis affects 75 million individuals in the United States, Europe, and Japan combined, with postmenopausal women accounting for one-third of the population and the elderly being the majority. Furthermore, osteoporosis is expected to become much more severe in the future [8]. A concern in the future, as the world's population continues to age. In Finland, for example, the overall incidence rate of hip fractures nearly quadrupled between 1970 and 1991, indicating that the population as a whole is at risk. Also of note, the age-standardized incidence rates of hip fractures nearly quadrupled at the same time, which is a first in medical history. A hip fracture in Finland resulted in immediate expenditures of 47,336 Finnish kronor (FIM) per patient in 1991. Hip fracture patients are expected to account for 11-13 percent of all surgical beds in Finland by the year 2000, according to projections [9].
When it comes to osteoporosis, there are several hereditary, physical, hormonal, and dietary variables that might be addressed [10]. Increased bone resorption in proportion to bone production, decreased bone mineral density, decreased trabecular bone volume, and, as a result, poorer mechanical properties of bone, which increases the risk of bone fractures, are typical signs of the osteoporotic stage of life. Osteoporosis is a disease that affects both men and women as they get older. Among women, however, estrogen shortage caused by the loss of ovarian function during menopause or following surgical ovariectomy causes the most significant changes in bone metabolism. The achievement of peak bone mass and the following rate of bone loss are the most important factors in the pathophysiology of osteoporosis [11-13]. As a result, the primary methods for osteoporosis prevention are the preservation of peak bone mass throughout adolescence and adolescence, and the prevention of bone loss after menopause and age $[14,15]$.

\section{Peak bone mass}

Peak bone mass refers to the greatest level of bone mass that an individual has reached as a result of normal growth and development [16]. The majority of people reach this stage throughout their first two to three decades of life (Bonjour., et al. 1991). Several factors, including diet, behavior, the environment, and mechanical stress, have been found to influence peak bone mass, with hereditary factors being the most important [17]. Recent twin studies have shown that hereditary variables may account for as much as 80 percent of the variation in bone mineral density in young people [18]. This information, however, is lacking in terms of understanding the proportional contribution of these variables to peak bone mass concerning other determinants [19]. Numerous hypothesized genes, including those affecting the metabolism of osteocalcin and type 1 collagen, have been proposed as being responsible for poor bone density. It is essential to have an adequate calcium intake in addition to polymorphisms linked with the vitamin D receptor gene and with the estrogen receptor gene of the dietary components involved. An appropriate calcium diet is required for the development and maintenance of a normal skeleton in children [20]. Furthermore, it has been demonstrated that calcium supplementation at amounts higher than the average daily consumption can enhance peak bone mass in children and adolescents. Calcium insufficiency, on the other hand, is considered to be present in the majority of teenage girls, most possibly across the whole world population [20]. In the United States, the recommended dietary intake for girls between the ages of 11 and 18 is $1200 \mathrm{mg}$ per day. However, a large number of recent clinical investigations [20] have demonstrated that this suggested amount is insufficient to ensure maximum skeletal buildup throughout time. Caution has been ad- 
vised in recent consensus conferences that daily calcium allowances between 1400 and $1500 \mathrm{mg} / \mathrm{d}$ be followed. Additionally, proper general nutrition, which includes adequate intakes of vitamins $\mathrm{D}, \mathrm{B} 6, \mathrm{~B} 12$, and $\mathrm{K}$, is essential for achieving maximum peak bone mass. A sufficient amount of physical activity is related with higher bone density levels in teenagers, according to recent research. Furthermore, it has been discovered that the bone density values of the dominant arm or leg in different groups of teenage athletes have grown as a result of their training. However, excessive physical activity in young women, which results in a reduction in body weight and decreased ovarian function, may result in a reduction in bone mass. Normal skeletal growth necessitates a normal endocrine state, which includes the functioning of the pituitary, adrenal glands, thyroid, and gonadal glands. Currently, an acquired gonadal hormone deficit, which is observed in many young women with eating disorders and is a specific concern in many western nations, is becoming more prevalent [21]. In addition, several lifestyle variables, such as smoking and alcohol addiction [21] may harm peak bone mass. Systemic disorders, such as hyperthyroidism and primary hyperparathyroidism, as well as medicines, such as excessive exposure to glucocorticoids, can all contribute to low peak bone mass in some people [22].

\section{Bone loss}

The skeleton of an adult is continuously being rebuilt. Achieving normal homeostasis involves maintaining a balance between bone resorption and bone production, which is primarily regulated by hormonal and mechanical mechanisms. Among the activities that remodeling is considered to enable are the maintenance of bone's load-bearing capability by avoiding and repairing microscopic structural damage, as well as the support of calcium homeostasis and hematopoiesis, among others. Bone remodeling is carried out by transient anatomic structures known as basic multicellular units, which are responsible for bone formation [23]. Remodeling is triggered by the differentiation of osteoclastic stem cells into mature multinucleated osteoclasts, which is the first step in the process. One phase of bone resorption is characterized by a tight attachment of activated osteoclasts to the bone, followed by actual bone absorption during which solubilization of hydroxyapatite crystals is accomplished by acidification of the resorption lacuna, followed by degradation of the organic bone matrix by lysosomal hydrolases and collagenases Mononuclear cells prepare the resorption lacunae for bone formation during the reversal phase of the cell cycle [24]. Bone production begins with the activation of preosteoblasts, which then develop into osteoblasts, which release bone-matrix proteins to form the organic matrix, which is eventually mineralized, as the first step in the process. During the remod- eling process at each remodeling site, bone resorption and bone creation are synchronized, with locally produced growth factors and cytokines functioning as mediators of the process [25]. It is believed that the reduction in bone mass is caused by an imbalance between the quantity of mineral and matrix that is removed and then absorbed into each resorption cavity 25). Once it has reached its maximum, bone mass begins to diminish gradually in both men and women, as a normal component of the aging process. When compared to women, bone loss in males and premenopausal women is relatively moderate, and it is largely caused by the progressive thinning of the trabecular plates and cortical bone, which is caused by the agerelated decrease in the quantity of matrix generated by the osteoblasts. Type II osteoporosis has been identified as the most common form of age-related osteoporosis. In addition to poor bone production, it is characterized by secondary hyperparathyroidism, which is caused by an age-related reduction in calcium absorption and results in higher bone turnover. Inadequate bone synthesis at the cellular level results in higher bone turnover, which in turn results in more bone loss. Increased intestinal resistance to 1,25-dihydroxy vitamin D3 and a reduction in the number of intestinal vitamin D receptors with age are likely to be responsible for the reduced calcium absorption seen in this study. As well as this, aging is linked with a decrease in the levels of growth hormone and insulin-like growth factor-1 [26], which may help to explain some of the variability in calcium homeostasis and bone turnover (for a review, see Blumsohn and Eastell 1995). Nutritional inadequacies and a lack of physical exercise are two other variables that have been reported to have an impact on bone turnover as people age. Following menopause, bone loss proceeds at a significantly faster rate in women. This is associated with an increase in bone turnover induced by estrogen deprivation, with bone resorption surpassing bone creation [26]. Following the cessation of ovarian function, bone marrow progenitors of both osteoclast and osteoblast origin develop more rapidly (for a review, see Manolagas and Jilka 1995), resulting in increased activation frequency of new basic multicellular units as well as an imbalance between bone resorption and formation at each remodeling unit, with the former exceeding the latter. With its vast surface area and thin network structure, cancellous bone is particularly vulnerable to this type of disruption. Consequently, there is a total loss of certain trabecular plates and considerable disruption of the trabecular lattice, with the trabeculae becoming more widely spaced and trabecular connection being decreased as a result of this process [27]. Per biomechanical principles, this leads to a decrease in bone strength that is disproportionately higher than the decrease in bone mass. Estrogen controls bone remodeling by regulating the synthesis of cytokines and growth factors by the bone marrow and bone cells, among other 
mechanisms. The capacity of estrogen to inhibit osteoclast generation and activation of mature osteoclasts, as well as its ability to induce apoptotic osteoclast death, appears to be responsible for its bone sparing action. On the other hand, a rise in the levels of interleukin-1 and tumor necrosis factor produced by estrogen deprivation has been postulated as a causal role in rapid bone loss. Type I osteoporosis has been described as osteoporosis that occurs after menopause. It is characterized by reduced parathyroid hormone secretion as well as decreased 1,25 hydroxyvitamin D3 synthesis, which results in decreased calcium absorption as a result of these changes. Postmenopausal osteoporosis is most likely not caused just by menopause, but also by several other variables that are present in some of these women and that worsen and extend the fast period of bone loss caused by estrogen deprivation. These additional factors include Many such variables, such as deficiencies in osteoblasts, which impede their capacity to enhance bone production to compensate for the increased bone resorption, may be present. additionally to a genetic predisposition for a certain type of cytokine release [28].

\section{Ziziphus}

Ziziphus mauritiana, commonly known as Jujube, Chinese Apple, Indian plum, and permeate (Anguilla), is a tropical fruit tree species that is a member of the Rhamnaceae family. It is native to Southeast Asia. Z. mauritiana is a spiny, evergreen shrub or small tree that grows up to $15 \mathrm{~m}$ tall and has a trunk $40 \mathrm{~cm}$ or more in diameter, a spreading crown, stipular spines, and numerous drooping branches. It is native to the island of Mauritius. There is a lot of variation in the form and size of the fruit. A variety's shape might range from oval to obovate to oblong to round, and its length can range from 1-2.5 in (2.5-6.25 cm) depending on the variety. The flesh is white and crisp, with a slight crunch. Despite being a little underripe, this fruit is rather juicy and has a nice scent even little underripe. The skin of the fruit is smooth and shiny, and it is thin yet tightly packed. It is most often found in tropical and sub-tropical climates around the world. Originally from India, it has now been widely naturalized throughout the tropical world, from Africa to Afghanistan and China, as well as via Malaysia and into Australia and parts of the Pacific [1]. This plant may grow in thick stands and become invasive in some locations, including Fiji and Australia. In Northern Australia, it has become a significant environmental issue due to its ability to pollute groundwater. It is a fast-growing tree with a medium lifetime that may grow up to 10-40 ft (3-12 $\mathrm{m})$ in height in a short period. [29].

\section{Botany}

It is a medium-sized tree that grows stronger and has a rapidly growing taproot, which is an essential adaptation to dry conditions. In stature, the species can range from a bushy shrub 1.5 to $2 \mathrm{~m}$ tall to a large tree 10 to $12 \mathrm{~m}$ tall, with a trunk diameter of around $30 \mathrm{~cm}$, depending on the variety. $Z$. mauritiana can grow upright or wide-spreading, with elegantly falling thorny branches, zigzag branchlets, thornless or set with small, sharp straight or hooked spines, or it can be thornless and thornless with zigzag branchlets and thornless.

Alternate, ovate or oblong elliptic with rounded apex, with 3 depressed longitudinal veins at the base of the leaves. The flowers are small and white. A fine tooth is present at the border of the leaves, which are around 2.5 to $3.2 \mathrm{~cm}$ long and 1.8 to $3.8 \mathrm{~cm}$ broad. The upper side is dark green and glossy, while the underside is pubescent and pale green to grey-green, depending on the variety. The $Z$. Mauritania's leaves can be either evergreen or deciduous, depending on the environment in which it grows.

The blooms are small, yellow, and 5-petaled, and they are generally seen in groups of two or three on the leaf axils. Inflorescences and fruits are white or greenish-white, 2-3 cm long, with an edible white pulp enclosing a 2-locular pyrene, while the flowers are white or greenish-white.

Within three years of planting, this fast-growing tree begins to produce fruit. The fruit is a delicate, delicious drupe with a diameter of $2.5 \mathrm{~cm}$, however with skillful culture, the fruit may grow to be $6.25 \mathrm{~cm}$ long and $4.5 \mathrm{~cm}$ wide, as well as $6.25 \mathrm{~cm}$ long and $4.5 \mathrm{~cm}$ broad. The shape can be oval, obovate, round, or oblong; the skin can be smooth or rough, glossy, thin but tough, and it can be smooth or rough, shiny, thin but tough. Even on the same tree, the fruit ripens at different periods of the year. Fruits are initially green, but as they mature, they turn yellow. In its fully ripe state, the fruit is crimson in color and soft and juicy in texture, with wrinkled skin and a nice scent. When the fruit is fully ripe, its flavor is both sweet and sour. The texture and flavor of the flesh are also evocative of apples. When the fruit is underripe, the flesh is white and crunchy, and the flavor ranges from acid to subacid to sweet. Fully ripe fruits are less crisp and more mealy in texture; overripe fruits are wrinkled, with flesh that is buff in color, mushy, spongy, and musky in flavor. When the fruit is overripe, the scent is initially apple-like and pleasant, but when it is underripe, it becomes very 
musky. There is a single, hard, oval or oblate, rough center stone that holds two elliptic, brown seeds that measure $1 / 4$ inch $(6 \mathrm{~mm})$ in length and $1 / 4$ inch $(6 \mathrm{~mm})$ in width [30].

\section{Ecology}

Z. mauritiana is a robust tree that can withstand high temperatures and thrives in dry circumstances, with annual rainfall ranging from 6 to 88.5 in (15-225 cm) on average. Ber trees have become naturalized in Fiji, and they may be seen growing in roadside ditches and agricultural fields, generally at sea level but rarely up to an elevation of about 600 meters. Furthermore, it grows well on laterite, medium-dark soils with adequate drainage, as well as on sandy, gravelly, alluvial soils of dry riverbeds, where it is a robust spontaneous grower. It may be found growing on a broad variety of soil types in Australia, including cracked clays, sodic soils, and deep alluvial soils, in the tropics and subtropics, where the average annual rainfall is between 470 and $1200 \mathrm{~mm}$. In the drier sections of this range, it thrives in riparian areas, which is where it originated [3]. Commercial production typically covers an area of up to $1000 \mathrm{~m}^{2}$. Trees do not perform well at this altitude, and agriculture becomes less cost effective as a result. Strong resistance to both waterlogging and drought exists in the tree, which may be found growing in places where annual rainfall ranges from 125 to 2,225 millimeters but is more common in areas where annual rainfall ranges from 300 to 500 millimeters. Wild trees may be found in China and India up to a height of 5,400 ft. in elevation $(1,650 \mathrm{~m})$. Indian shade temperatures range from 7 to 13 degrees Celsius, with the highest temperature reaching 50 degrees Celsius. According to research, this plant thrives on alkaline soils with $\mathrm{pH}$ values as high as 9.2. Although deep sandy loam to loamy soils with neutral or slightly alkaline $\mathrm{pH}$ is regarded ideal for growth [5] in India, the tree grows best on sandy loam soils that are neutral to slightly alkaline in $\mathrm{pH}$ [6]. It is estimated that there are more than 90 different cultivars of mango in India, each with its characteristics such as tree habit and leaf shape as well as fruit form and size, flavor, keeping quality, and fruiting season. In the encyclopedic Wealth of India, eleven cultivars are listed as important: 'Banarasi (or Banarsi) Pewandi', 'Dandan', 'Kaithli' (also known as 'Patham'), 'Muria Mahrara', 'Narikelee', 'Nazuk', 'Sanauri 1', 'Sanauri 5', The skin of the majority of them is smooth and greenish-yellow to yellow. Reproductive Biology is a branch of biology that studies reproduction. Some cultivars reach anthesis first thing in the morning, whilst others do so later in the afternoon. The blooms have a protandrous fragrance. As a result, the fruit set is dependent on cross-pollination by insects drawn to the flower's smell and nectar. The pollen of the Indian jujube tree is thick and has heavy inconsistency. Although it is not airborne, honeybees are responsible for spreading it from blossom to flower. Aphids and other insects pollinate the blooms, which means that in the wild, the trees do not set fruit since they are not pollinated by themselves. Ber can be propagated through seeds, seedlings, direct planting, root suckers, and cuttings, among other methods. Ber seeds are distributed by a variety of species, including birds, native animals, livestock, feral pigs, and people, who consume the fruit and eject the seeds after eating it. Seeds may be kept alive for up to 212 years, although the rate of germination decreases as the seeds get older. The occurrence of cross-incompatibility necessitates the pairing of cultivars to get optimal fruit set; certain cultivars yield excellent crops when grown parthenocarpically [31,32].

Propagation

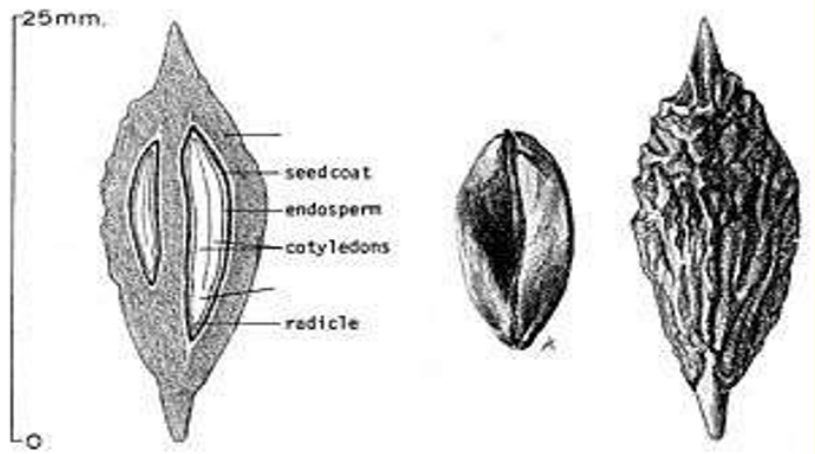

Figure 1

The inside anatomy of the jubilee seed is shown in figure 1 . It is beneficial to receive pretreatment. The storage of the seed for 4 months to allow it to after-ripen enhances the germination rate of the seed. Seed germination is restricted by the hard stone, and germination is accelerated by breaking open the shell or extracting seeds from the stone. In the absence of pretreatment, seeds typically sprout in six weeks, whereas extracted seeds germinate in one week or less. It is possible to produce seedlings that will be utilized as rootstock from seed. Seed germination is enhanced by soaking seeds in sulfuric acid, according to several studies. By properly breaking the endocarp, it is possible to reduce the germination time to 7 days or less. Ber seedlings are not tolerant to transplantation; thus, the best options are to sow the seeds immediately in the field or to use polythene tubes put in the nursery bed to protect the seedlings. Seedlings are ready for budding in 3 to 4 months after thy are planted. In addition, seedlings from wild cultivars can be transformed into better cultivars by top working and grafting, which are both rather simple processes. Nurseries are 
used for large-scale seedling multiplication and graft production, as well as for a variety of other purposes. In addition, the seedlings should be exposed to direct sunlight. The seedlings may need as much as 15 months at the nursery before they can be planted in the field [4].

Indian scientists have developed conventional propagation strategies for the development of Ber colonies. Budding is the most straightforward technique of vegetative propagation, and it is most commonly employed for better cultivars. The use of several sorts of budding procedures has been attempted, with the most effective being ring-budding and shield-budding, respectively. Ber rootstock is often derived from wild forms of the plant. The most common are Z. rotundifolia in India and Z. Spina Christi in Africa, with Z. rotundifolia being the most common worldwide [33].

\section{Season and harvesting}

In the backdrop, you can see fully ripe red-colored Indian jujube fruits that are being sold in Delhi. Once plants reach a height of around 1 meter, they can produce seeds for reproduction. It may take up to 8 years for wild-growing plants in northern Australia to reach this size. Upon reaching a height of around 1 meter in Australia, plants living in their natural environment can reproduce and produce seeds. Only around five fruits are produced every season by plants between one and two meters in height, according to research. Large plants ( $>5 \mathrm{~m}$ tall) have the potential to yield 5000 or more fruits in a single growing season. Some varieties mature as early as October in India, while others ripen from mid-February to midMarch, and still, others ripen in March or from mid-March to the end of April. In the Assiut Governorate, there are two harvests every year, the first of which is harvested in the early spring and the second of which is harvested in the fall. In India, the trees blossom from July to October, and the fruits are developed shortly thereafter. The fruits are fully mature between February and March, and in certain areas, a second harvest is produced in the fall as well. Picking is done by hand from ladders, and around $110 \mathrm{lbs}$ (50 kg) of produce is gathered every day. The fruits that are still on the tree are shaken to the ground. Picking fruits directly from the tree is limited to fully ripe fruits only. They are transported in open bags to prevent the growth of bacteria. In India, seedling trees produce between 5,000 and 10,000 tiny fruits each year. Superior grafted plants have the potential to produce as many as 30,000 fruits. The finest cultivar in India, with fruits typically weighing $30 \mathrm{lbs}$ (66 kg) every pound ( $66 \mathrm{~kg}$ per kilogram), produces $175 \mathrm{lbs}$ (77 kg) of fruit each year. Fruit size and yield are both increased as a result of special cultural treatment [34].

\section{World production and yield}

The dry and semi-arid regions of India are the major production areas for Indian jujube, which is grown across the nation. A total of 0.9 million tons of grain was produced across an 88,000-hectare area between 1984 and 1995, thanks to improved cultivars that increased yields. The crop is grown in countries such as Pakistan, Bangladesh, and parts of Africa, in addition to the United States. The production of fresh fruit per tree in northern India ranges from 80-200 kg per year, depending on the type, when the trees are at their optimum producing age of 10-20 years.

Uses

The fruit may be eaten raw or pickled, and it can also be used to produce beverages, such as smoothies. It is a rich source of nutrients and contains a significant amount of vitamin C. The only guava is more popular in terms of popularity, and it rates considerably higher than citrus or apples in terms of popularity. In India, ripe fruits are often consumed raw, although they can also be cooked or roasted if they are available. Somewhat underripe fruits are candied using a method that involves pricking and immersing fruits in a salt solution for some time. It is necessary to sun-dry ripe fruits to keep them fresh longer, and a powder is created for usage when the fruits are not in season. In terms of nutritional value, depending on the type, it contains between 20 and $30 \%$ sugar, up to 2.5 percent protein, and 12.8 percent carbohydrates. Fruits can be consumed in a variety of forms, including dried, candied, pickled, juiced, and turned into ber butter, among other things. The fruits are commonly used to stupefy fish in Ethiopia, where it is a customary practice to do so. It is widely believed that the leaves are nutritious in nature since they are readily devoured by camels, cattle, and goats. In India and Queensland, where they are considered as such, honeybees use the flowers as a tiny source of nectar for their young. Colorless and with a mild flavor, the honey is a sweet treat. Berwood has a reddish color and a fine grain pattern, and it is hard, strong, fine-grained, fine-textured, durable, and long-lasting. Ber wood has a reddish hue and a fine grain pattern. Most frequently, it is used to construct agricultural equipment, but it has also been used to line wells, to construct bedstead legs, to construct boat ribs, and to construct other objects. The branches are used as a framework in the construction of dwellings, and the wood makes excellent charcoal, with a heat capacity of about 4,900 kcal per kilogram of wood. Wood is also used to make furniture. Furthermore, this species is frequently used as firewood in a range of climates due to its versatility. Tropical Africa is home to a variety of applications for the flexible branches, including the construction of retaining bands around conical thatched roofs and the construction of thorny corral walls to keep animals contained. 


\section{Z. mauritiana bark}

The fruits are used to treat wounds and ulcers, as well as lung illnesses and fevers. They are also given to those suffering from indigestion and biliousness when combined with salt and chili peppers. The dried ripe fruit has a slight laxative effect when consumed. The seeds have a calming effect and are eaten, often in conjunction with buttermilk, to relieve nausea, vomiting, and stomach discomfort associated with being pregnant. They are used to check for diarrhea and to apply poultices to wounds. They are used topically to rheumatoid joints after being mixed with oil. The leaves are used as poultices and are beneficial in the treatment of liver problems, asthma, and fever. They are also used in conjunction with catechu when an astringent is required, such as on wounds. The bitter, astringent bark decoction is used to treat diarrhea and dysentery, as well as gingivitis and gingivitis-like symptoms. A paste made of bark is put directly on wounds. The root has purgative properties. Infusions of the root are used as a febrifuge, taenicide, and emmenagogue, and the powdered root is used topically to wounds. Gout and rheumatism are believed to be alleviated by drinking the juice from the root bark. Large amounts of the bark or root may be poisonous. It is possible to use an infusion of the blossoms as an eye lotion. All of the primary biodiesel standards in the United States (ASTM D 6751-02, ASTM PS 121-99), Germany (DIN V 51606), and the European Union (EN V 51606) are satisfied by the fatty-acid methyl ester of Z. mauritiana seed oil (EN 14214). This plant produces $4.95 \mathrm{~kg}$ oil per tree or $1371 \mathrm{~kg}$ oil per hectare, and it may be grown in dry or semi-arid locations because to its drought resilience. It can also be grown in tropical climates [35].

\section{Pests and diseases}

Fruit flies are the jujube's most formidable foe. When fruit is sweet and big, the flies favor it, and a significant portion of a fruit tree's harvest may be attacked on the neighbor's trees, which grow smaller and less-sweet fruit and only a tiny fraction of which will be attacked. It has been discovered that treatment of the ground underneath the tree helps to lower the occurrence of larvae. It is feasible to keep bug populations under check with frequent pesticide application. Caterpillars known for their appetite for leaves will attack the leaves.

Twig galls that look like scales occur due to mites, causing growth setbacks and decreasing fruit production. A little caterpillar is one of the less threatening bugs. Meri arches scrods is an insect that dwells in fruit. The parasitic vine covers the tree in its shadow. Powdery mildew results in leaves dropping off and fruit loss, although effective management can be achieved. A few, minor illnesses like sooty mold, brown rot, and leaf spot are on the list.
Leafspot, caused by the fungus Cercospora spp. and the parasite Isariopsis indica var. zizyphi, appears on many plants. It was discovered in 1973 that mycoplasma-like organisms had produced a witches' broom illness in jujube plants at Poona University. Transmitted via grafting or budding infected scions onto healthy $Z$. mauritiana seedlings, it was discovered to have been spread. Leaf rust, which has different degrees of severity, is seen on all commercial cultivars in Punjab, a province in Pakistan. Fungi may be present in the storage, which might spoil the fruit. Fusarium spp., Nigrospora oryzae, Epicoccum nigrum, and Glomerella cingulate are all responsible for rotting fruit [36].

\section{Material and Methods}

\section{Materials}

\section{Animals}

It was decided to utilize 112 adult female non-pregnant albino rats, each weighing between 250 and 350 grams, for this experimental research. The animals were 90 days old at the time of slaughter. Throughout the trial, twelve rats died, and only 100 rats made it until the end. The animals were fed on an ad libitum basis and were given unlimited access to water. They were kept in cages under rigorous care and cleanliness standards to maintain normal and healthy circumstances for the animals.

The animals Were divided into four main groups

Group I: Group received the antiestrogen drug, Tamoxifen (Tam), orally, The following subgroups were included in this group:

- Subgroup I: Consisting of ten rats who took the medication orally for four weeks before being sacrificed (I rat died).

- Subgroup II: This group consisted of ten rats who were given the medication orally for eight weeks before being killed (I rat died).

- Subgroup III: This group consisted of 10 rats who were given the medication orally for 12 weeks before being killed. Subgroup IV: Comprised 10 rats that were left to recover for 4 weeks after stopping the drug to evaluate the degree of reversibility of changes induced by the drug i.e. they were sacrificed after 16 weeks (I rat died).

Sum of the 2 groups: $37+15=52$ rats.

Group II: Control group which received only a vehicle of distilled water consisted of 16 rats. Only one rat died during the experiment. Four of these animals were sacrificed with each experimental subgroup receiving the drug and 3 rats were sacrificed with the recovery group. 


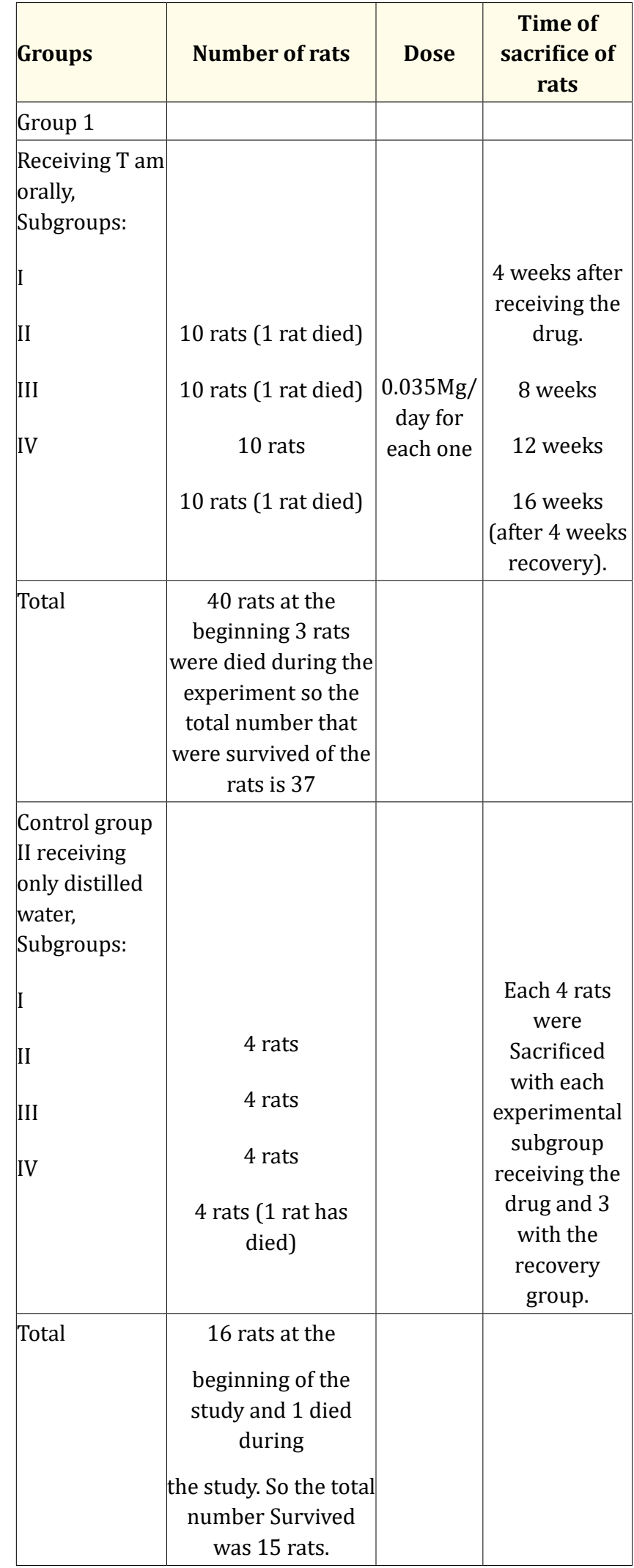

Table 1: Rats receiving Tamoxifen and their controls; the number of rats, dose of drug received and time of their sacrifice.
Group III: Bilaterally ovariectomized rats. This group comprised the following subgroups:

- $\quad$ Subgroup I: Comprised 10 rats which were sacrificed 4 weeks following ovariectomy (OVX) (5 rats died).

- $\quad$ Subgroup II: Comprised 10 rats which were sacrificed 8 weeks following OVX (1 rat died).

- $\quad$ Subgroup III: Comprised 10 rats which were sacrificed 12 weeks following OVX.

- Subgroup IV: Comprised 10 rats that received Tam orally for 4 weeks following the 12 weeks OVX period and then were sacrificed.

Group IV: Control sham-operated group. This group comprised the following subgroups:

- $\quad$ Subgroup I: Comprised 4 rats which were sacrificed 4 weeks after the sham operation (2 rats died).

- $\quad$ Subgroup II: Comprised 4 rats that were sacrificed 8 weeks after the sham operation.

- Subgroup III: This group consisted of four rats that were killed 12 weeks following the sham surgery.

- Subgroup IV: This group consisted of four rats that were killed 16 weeks after the sham surgery was performed. It was necessary to maintain the OVX animals on the same food intake as their respective sham-operated controls in the same dietary group "pair feeding" to prevent the hyperphagia that is associated with OVX.

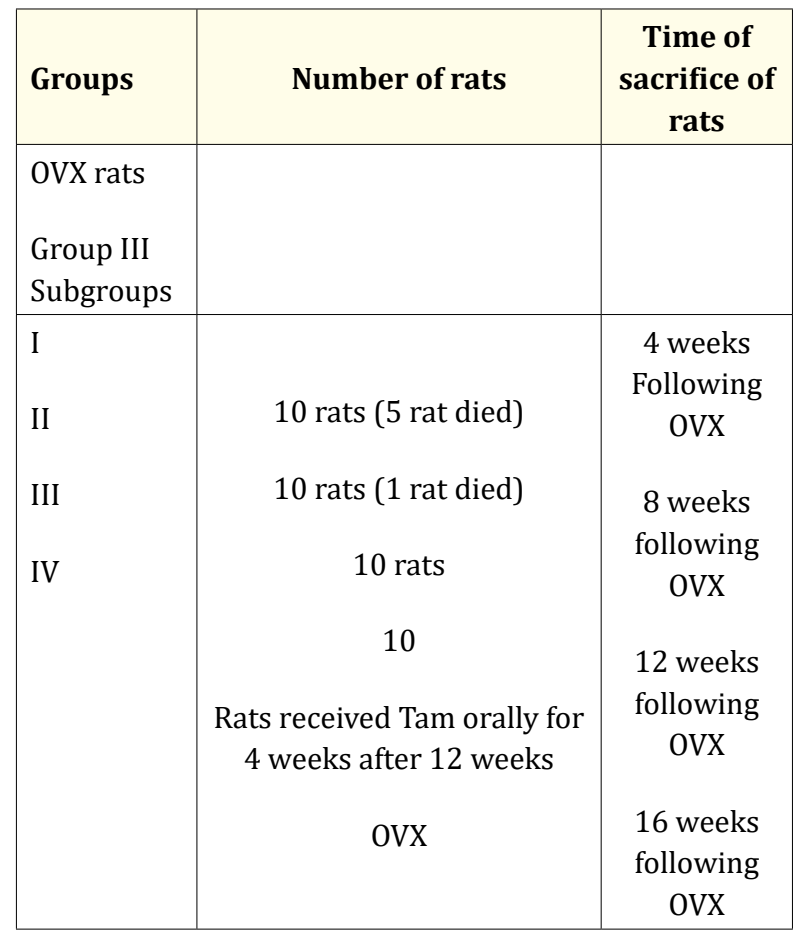




\begin{tabular}{|c|c|c|}
\hline Total & $\begin{array}{c}40 \text { rats at the beginning of } \\
\text { the study and } 6 \text { died during } \\
\text { the experiment so the total } \\
\text { number } \\
\text { Survived was } 34 \text { rats. }\end{array}$ & \\
\hline $\begin{array}{l}\text { Operated } \\
\text { rats } \\
\text { Subgroups } \\
\text { I } \\
\text { II } \\
\text { III } \\
\text { IV }\end{array}$ & $\begin{array}{l}4 \text { rats (2 rat died) } \\
4 \text { rats } \\
4 \text { rats } \\
4 \text { rats }\end{array}$ & $\begin{array}{c}4 \text { weeks } \\
\text { after sham } \\
8 \text { weeks } \\
\text { after sham } \\
12 \text { weeks } \\
\text { after sham } \\
16 \text { weeks } \\
\text { after sham }\end{array}$ \\
\hline Total & $\begin{array}{l}16 \text { rats at the beginning of } \\
\text { the study and } 2 \text { died during } \\
\text { the experiment so the total } \\
\text { number Survived was } 14 \\
\text { rats. }\end{array}$ & \\
\hline $\begin{array}{l}\text { Sum of the } \\
2 \text { groups: }\end{array}$ & $34+14=48$ rats & \\
\hline
\end{tabular}

Table 2: Bilaterally ovariectomized rats and their sham controls; many rats and time of their sacrifice.

The drug

The medication utilized in this trial was Tamoxifen citrate (Amrita pharmaceutical business Egypt), which was administered in the form of $10 \mathrm{mg}$ tablets to the participants.

$0.035 \mathrm{mg} /$ day for each animal was obtained by crushing the pills into a powder and dividing the powder into equal parts.

Each dosage was dissolved in distilled water and administered intragastrically through a tube in the stomach. Following the approach of Paget and Barnes, this dose was calculated by extrapolating it from the proposed human therapeutic dose (1964).

\section{Methods}

Operations

- Ovariectomy

- Sham operation

\section{Ovariectomy (OVX)}

All of the rats were put in the supine posture and given a little amount of ether anesthesia.
The 40 rats were subjected to bilateral ovariectomy using a dorsal approach (Waynforth, 1980) and a midline incision, after cleaning of the front abdominal wall with povidone-iodine (Betadine). The abdomen was examined for the presence of a bicornuate uterus and then along with the two uterine horns towards the aries, after which the ovarian pedicle and vasculature were ligated with vicryl 30 synthetic, absorbable, polyglactin sutures (Ethicon business, America) and the ovaries were removed.

When the incision was closed, the powdered aqueous penicillin (Epico pharmaceutical business, Egypt) was dissolved in distilled water and injected intraperitoneally. The wound was then closed using the same suture material as was used for the first closure. Following a procedure, Garamycin cream was used topically as a wound dressing for a total of 7 days to minimize the risk of wound infection, which is most likely to develop $5-7$ days after the operation.

\section{SHAM operation}

The abdominal cavities of control rats were opened, but their ovaries were kept intact so that they could be subjected to the same amount of stress as the rats that had their abdominal cavities opened. The same sterile measures were used and the same method of wound closure was used as in the ovariectomy group.

\section{Study of the morphological changes}

Rats were slaughtered by ether anesthesia followed by an abdominal opening with a midline incision at the above-mentioned periods after receiving OVX. Using formalin-based fixation, the left tibiae and femora from each animal were cleansed of soft tissue and put in 10 percent phosphate-buffered formalin for 24 hours. Then they were decalcified with 10\% EDTA (ethylene diamine tetraacetic acid) for 4 days to remove the calcium deposits. In each animal, the right tibia and femur were defleshed and put in $10 \%$ phosphate-buffered formalin for 24 hours before being analyzed. The bone specimens were subsequently dried in ethanol and implanted undecalcified in methylmethacrylate to demonstrate the presence of minerals in bone tissue. It is impossible to prevent the mineralized tissue from collapsing when cutting it with a soft embedding medium, such as paraffin wax. Acrylic resins, particularly methyl methacrylate, are probably the most helpful as a supporting medium for the undecalcified bone because of their flexibility. With the use of this embedding media, it is feasible to get sections of mineralized bone that have not been damaged, allowing mineral density and defects to be examined in conjunction with the osteoid It is common practice to utilize these resins in the preparation of material for electron microscopy experiments (Bancroft and Ste- 
vens, 1996). Using a tungsten carbide knife (Leica pharmaceutical business, Germany), which has blades with abrasive cutting edges that are diamond-impregnated, sections were cut out of the samples to be analyzed. To stain the calcified sections, a modified Masson Goldner's trichrome solution was used (Baron., et al. 1983). The uterine specimens are a different story. They were discovered just four weeks after the OVX procedure, not later. It was necessary to expose the uteri by moving the viscera to one side, and they were only discovered after much difficulty due to their severe atrophy. The uteri were extracted, defatted, and treated in preparation for inspection under light and electron microscopes, respectively. Preparation of paraffin blocks for light microscopic studies began with the fixation of uterine horn mid-portions in Bouin's solution, followed by dehydration in ascending grades of alcohol, followed by clearing in benzene, followed by embedding in hard paraffin with a melting point of $62^{\circ} \mathrm{C}$ for three hours, followed by the preparation of paraffin blocks. Five o'clock a.m. The rotator microtome was used to cut thick serial slices of the specimen (Leica pharmaceutical company, Germany) The identical specimens (tibiae and femora, as well as the uterine horns) were obtained from the Tam group and their controls, and they were submitted to the same processing techniques as those used for the OVX group and their sham controls (see Table 1).

All of the samples were collected during the estrous phase of the female reproductive cycle. This phase was determined using the vaginal smear method, which involved cleaning the rats' vagina with saline using a pipette as a washing solution. After being inspected microscopically, it was found that the samples were in their estrous phase if they exhibited scales and that they were in their diestrus phase if they showed nuclei. The Papanicolaou stain was used to determine which phase they were in.

The following strains were used

- H\&E stain.

- Masson's trichrome stain.

- Goldner's trichrome (Modified Masson) stain for undecalcified bone sections.

- Alcian blue/PAS for demonstration of acidic and neutral mucopolysaccharides.

- Papanicolaou stain for vaginal smears.

- Toluidine blue for semithin sections of uterine specimens.

- Lead citrate and uranyl acetate for ultrastructure of uterine epithelium.
Massons trichrome stain

(After Masson, 1929) (After Culling, 1974).

\section{Principle}

It is one of the multistage techniques Which aim at differentiating tissue structures such as collagen from muscle, in contrasting colors.

\section{Solutions}

Ponceau-acid fuchsin solution.

Equal volumes of $0.5 \%$ ponceau $2 \mathrm{R}$ in $1 \%$ acetic acid and $0.5 \%$ acid Fuchsin in $1 \%$ acetic acid.

- $\%$ aqueous phosphomolybdic acid.

- $\quad \%$ light green in 2\% acetic acid diluted 1: 10 With distilled water before use.

Technique: Sections were

- Taken to water.

- $\quad$ Differentiated, blued, and washed in water after staining the nuclei with an iron hematoxylin solution.

- $\quad$ Treated with the ponceau-acid fuchsin solution for 2-3 minutes.

- Washed in water and differentiated in the phosphomolybdic acid solution (usually for 5-15 minutes at room temperature). Washed well in water.

- Counterstained in light green solution for l minute.

- Washed, dehydrated, cleared, and mounted.

\section{Comments}

A significant factor in determining the effectiveness of this approach is the degree to which the phosphomolybdic acid differentiates the ponceau acid fuchsin. It is critical to keep the differentiation process going until the connective tissue is almost completely unstained.

The process of differentiation can be sped up by heating the solution to 56 degrees Celsius.

Results

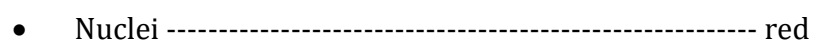

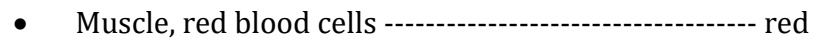

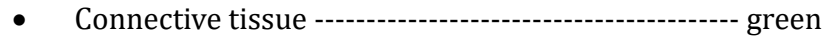


Goldner's Trichrome Stain (After Goldner, 1938) (After Bancroft and Stevenes, 1996).

\section{Principle}

This method is applied for staining undecalcified methacrylateembedded bone sections where the aim is chiefly to distinguish the mineralized bone from the osteoid. This method is a modification of Masson's trichrome stain.

\section{Solutions}

\section{Weigert's hematoxylin}

This is an iron hematoxylin in which ferric chloride is used as the mordant/oxidant. The iron and the hematoxylin solutions are prepared separately and are mixed immediately before use.

They are prepared as follows

Haematoxylin solutions

- Haematoxylin $1 \mathrm{~g}$

- Absolute alcohol $100 \mathrm{ml}$

- Iron solution $30 \%$ aqueous ferric chloride $-4 \mathrm{ml}$

- Hydrochloric acid $1 \mathrm{ml}$

- Distilled water $95 \mathrm{ml}$

This solution is filtered and added to an equal volume of the hematoxylin solution immediately before the stain is used. The mixture should be a violet color and must be discarded if it is brown.

Ponceau - fuchsin - Azophloxin solution

- Ponceau de xylidine $0.75 \mathrm{~g}$

- Acid fuchsin $-0.25 \mathrm{~g}$

- $\quad$ Acetic acid $-1.0 \mathrm{ml}$

Mix and add to $100 \mathrm{ml}$ distilled water

- Azophloxin $0.5 \mathrm{~g}$

- Acetic acid $-1.6 \mathrm{ml}$

Mix, and add to $100 \mathrm{ml}$ distilled water.

The final staining solution is made by mixing

- Ponceau - fuchsin solution $5-10 \mathrm{ml}$

- Azophloxin $2 \mathrm{ml}$

- $\quad$ Acetic acid solution (0.2\%) $88 \mathrm{ml}$
Light green solution

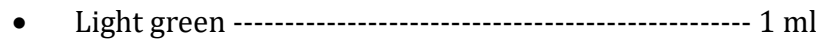

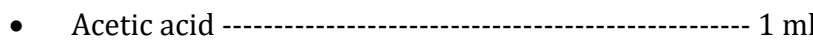

Mix, and add to $500 \mathrm{ml}$ distilled water.

Phosphmolybdic acid/orange G solution

- $\quad$ Phosphomolybdic acid .........................................3g

- Orange G.......................................................

Dissolve in $500 \mathrm{ml}$ of distilled water, and add a crystal of thymol.

Method: Section were

- Left in a solution of $90 \mathrm{ml} 80 \%$ ethanol and $10 \mathrm{ml} 25 \%$ ammonia for 1 hour.

- $\quad$ Rinse in water for 15 minutes.

- Stained in Weigert's hematoxylin for 1 hour.

- $\quad$ Rinse in water for 10 minutes.

- $\quad$ Rinse in distilled water for 5 minutes.

- $\quad$ Stained in final ponceau-fuchsin -azophloxin solution for 5 minutes.

- $\quad$ Rinse in $1 \%$ acetic acid for 15 seconds.

- $\quad$ Stained in phosphomolybdic acid/orange G solution for $20 \mathrm{~min}$.

- $\quad$ Rinse in $1 \%$ acetic acid for 15 seconds.

- $\quad$ Stained with light green for 5 minutes.

- $\quad$ Rinse in 3 changes of $1 \%$ acetic acid.

- $\quad$ Rinse in distilled water blotted dry, and mounted.

Results

- Mineralized bone..............................green

- $\quad$ Osteoid ...........................................orange/red

- $\quad$ Nuclei...................................................ed

Combined Alcain blue/PAS Technique (After Mowry, 1956) (After Culling, 1974).

\section{Principle}

Other ways may be used to discriminate between acid mucins and neutral mucins, but this is one of the most helpful. It also serves the purpose of demonstrating the presence of the majority of mucins in a single preparation. In practice, this means that a 
negative result, such as an alcian blue negative or a PAS negative, might be interpreted as indicating that a particular chemical is unlikely to be a min.

The logic behind this approach is that by first staining the acid musicians with alcian blue, the acid musicians will be rendered incapable of reacting with the following PAS solution. Follo wing the PAS procedure, only neutral mucins and carbs such as glycogen will stain red, excluding all other substances.

\section{Solutions}

- $1 \%$ alcian blue in $3 \%$ acetic acid

- $1 \%$ aqueous periodic acid

- Schiff's reagent :

- Basic fuchsin. $1 \mathrm{~g}$

- Distilled water. $.200 \mathrm{~g}$

- Potassium or sodium metabisulphite $2 \mathrm{~g}$

- Concentrated hydrochloric acid. $.2 \mathrm{~cm}^{3}$

- Decolorizing charcoal. $0.2 \mathrm{~g}$

Bring the distilled water to a boil, then turn off the heat (to avoid an excessive amount of effervescence) and add the basic fuchsin to taste. Combine the ingredients, cool to $50 \mathrm{0C}$, and then add the metabisulphite. Before adding the hydrochloric acid, combine the ingredients and allow them to cool to room temperature. Leaving it out overnight in the dark and at room temperature is recommended. Add charcoal the following day and let it in the dark for 12 minutes. After filtering, keep it at 40 degrees Celsius in a dark container.

With continued usage, it will be discovered that the initially colorless to the light yellow solution will gradually become pink due to the loss of sulfur dioxide, resulting in the restoration of the original fuchsin hue.

When this occurs, the solution should be thrown out immediately.

Technique: sections were

- $\quad$ Take to distilled water.

- $\quad$ Treated with the alcian blue solution for 5 minutes.

- Washed well in distilled water.

- $\quad$ Treated with the periodic acid solution for 2 minutes.

- Washed well in distilled water. Then washed in Schiff's reagent for 8 minutes.
- Washed in running water for 10 minutes.

- Differentiated and blued a fret staining the nuclei with hematoxylin solution.

- $\quad$ Dehydrated, cleared, and mounted.

Results

- Acid mucins -

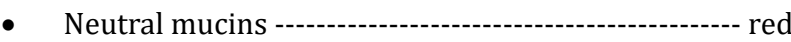

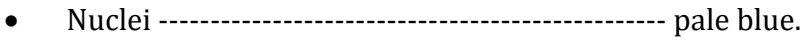

Papanicolaou stain (After Papanicolaou, 1942) (After Naib, 1976)

This method is very popular for the cytological examination of smears of the female reproductive tract.

Solutions

Orange G

- Orange G-- $0.5 \mathrm{~g}$

- Phosphotungestic acid $0.015 \mathrm{~g}$

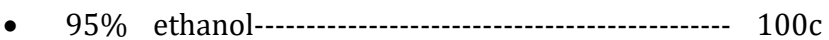
$\mathrm{m}^{3}$

EA 50

- $\quad 0.1 \%$ light green in 95\% ethanol -------------------45 $\mathrm{cm}^{3}$

- $\quad 0.5 \%$ eosin yellowish in $95 \%$ ethanol -------------45 $\mathrm{cm}^{3}$

- $\quad 0.5 \%$ Bismarck brown in 95\% ethanol --------------45 $\mathrm{cm}^{3}$

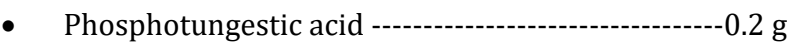

- Saturated aqueous lithium carbonate -------------1 drop.

Technique: Sections were

- $\quad$ Treated with $95 \%$ alcohol for 1 minute.

- $\quad$ Treated with $70 \%$ alcohol for 2 minutes.

- $\quad$ Rinsed with distilled water for 3 minutes.

- Treated with Harris, hematoxylin solution for 21/2 minutes $\stackrel{a}{ }$ Rinsed in tap water for 1 minute.

- $\quad$ Treated with $1 \%$ acid - alcohol for 3 seconds.

- $\quad$ Rinsed in tap water for 2 minutes.

- Treated with ammoniated water (distilled water to which are added a few drops of concentrated ammonia) for 1 minute.

- $\quad$ Rinsed in tap water for 3 minutes. 
- $\quad$ Treated with $70 \%$ alcohol for 2 minutes.

- $\quad$ Treated with $95 \%$ alcohol for 2 minutes.

- $\quad$ Stained with OG6 solution for 1 minute.

- $\quad$ Treated with 95\% alcohol for 1 minute.

- $\quad$ Stained with EA50 solution for $11 / 2$ minutes.

- $\quad$ Treated with $95 \%$ alcohol for $1 / 2$ minute.

- $\quad$ Treated with absolute alcohol for 3 minutes.

- $\quad$ Treated with xylene for 4 minutes.

- $\quad$ Treated with xylene and mounted.

Results

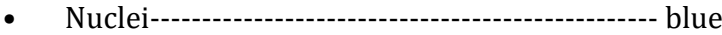

- $\quad$ Superficial (cornified) cells -------------------- pink

- Intermediate (non-cornified) cells -------------green

Preparation for electron microscopic studies

Principle

Because the wavelength of a voltage accelerated electron beam is relatively small, it has the potential to provide a significantly hi gher resolving power than that produced utilizing light rays in many applications.

This resulted in the development of the electron microscope, which uses an electric beam in stead of light beams to magnify objects.

After passing through the evacuated microscopic column, which has a v shaped tungsten filament as its cathode, the electrons are propelled towards a node that contains an aperture through which the electron beam travels before being focussed onto a specimen by condenser lenses.

Depending on the model, the electron microscope's power of magnification can be greater than 250000, and its resolving power can be better than 0.25 nanometers).

\section{Procedure for conventional EM studies}

\section{Fixation}

The tissue specimens were first prefixed by $3 \%$ glutaraldehyde freshly prepared from the were kept at room temperature for two hours then washed three times in the same buffer.
The specimens were then postfixed in $1 \%$ osmium tetraoxide in phosphate buffer (prepared from a stock of $4 \%$ osmium) for 2 hours at $4^{\circ} \mathrm{C}$.

N.B: Utmost care should be taken in handling osmium as it is a very potent fixative and fixes tissue rapidly even by inhalation of fumes. Therefore this procedure must be performed in the hood for safety measures

The specimen should turn brownish-black in color after postfixation with osmium to ensure proper penetration of the fixative the specimen were then rinsed three times in distilled water and prepared for dehydration.

\section{Dehydration}

Dehydration was accomplished at room temperature in graded ethanols (Adwick Co. Egypt) as follows:

- $30 \%$ ethanol for 5 minute.

- $\quad 50 \%$ ethanol for 5 minute.

- $\quad 70 \%$ ethanol for 5 minutes.

- $\quad 90 \%$ ethanol for 5 minute.

- $\quad 95 \%$ ethanol for 5 minute.

- $100 \%$ ethanol for 5 minute.

The last repeated step was twice for $10 \mathrm{~min}$. each.

The specimens were then treated in a mixture of volumes of ethanol $100 \%$ and propylene oxide $100 \%$ for $5 \mathrm{~min}$. The specimen was treated in $100 \%$ acetone for another $5 \mathrm{~min}$.

Most plastic embedding mixtures are not miscible with ethanol, therefore a final step of dehydration with propylene oxide (BDH EM grade) or acetone was performed.

N.B: the tubes should always be covered with a parafilm paper during dehydration to avoid evaporation of ethanol or acetone and dryness of the specimen which causes it to become brittle and on sectioning.

Infiltration and embedding

Most embedding mixtures are not miscible with ethanol. therefore acetone or propylene was used as the intermediate solvent.

Equal volumes of upon and acetone or (propylene oxide), left on the specimens for I hour, facilitated the infiltration of the resin. 
The hardness of the final block could be adjusted by the ratio of two mixtures (CatovsKy, 1981).

Solution (A)

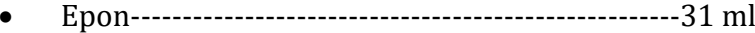

- Dodeceyl succinic anhydride DDSA-------------50 ml.

Solution (B)

- $\quad$ Epon$100 \mathrm{ml}$

- Methyl nadic anhydride $-89 \mathrm{ml}$.

The usual ratio used was $7 \mathrm{ml}$ of $\mathrm{B}$ and $3 \mathrm{ml}$ of $\mathrm{A}$ which were mixed together with the final addition of 6 big drops of the activator DMP-30.

The fixed tissue specimens were disposed of in the beam capsule where a small drop of upon was placed at its bottom. A label was put in each capsule for identification.

\section{Polymerization}

- It was done at $60^{\circ} \mathrm{C}$ for 24 hours.

\section{Ultramicrotomy}

The beam capsule was stripped off by making two longitudinal cuts in the capsule with a sharp razor in order to obtain the sample block.

Glass knives were made from glass bars $25 \mathrm{~mm}$ thick (LBK) ON an (LBK) knife maker. The sample block was fixed in the holder of the LBK ultramicrotome. Trimming was done to obtain a pyramid with a trapezoid surface having two parallel sides.

A plastic boat was attached to the knife and the formed trough was filled with distilled water for the cut sections to float on. First, semi-thin or thick sections were cut, collected on glass slides, and left to dry on a hot plate. They were then stained with toluidine blue $1 \%$ solution for I minute on the hot plate $\left(60-120^{\circ} \mathrm{C}\right)$. The sections were quickly rinsed with distilled water. After drying, these solutions were examined by the oil-immersion power of a meta microscope to ensure the presence of the required specimens in the cut sections.

Ultrathin sections (60-100 $\mathrm{nm}$ thickness) with a silver or golden color (Dawes, 1980) were cut by a new glass knife to make a ribbon of sections. violet or green colored sections were discarded as their color indicated that they were thicker. They were exposed to chloroform vapors to remove any folds or wrinkles.
They were then picked from the distilled water on copper grids (BDH EM grade) either with a Platinium loop or applying the grid directly on the ribbon. The grids were left to dry on filter paper in a petri dish.

\section{Staining of the grids}

The grids were stained by a double staining technique uranyl acetate followed by lead citrate solutions. Saturated aqueous solutions of uranyl acetate were used for moderate staining. The dissolution of this stain in water was very slow. Uranyl acetate was more soluble in methanol than in water or ethanol. A 7\% solution of this stain in 50\% methanol was used (Hayat, 1986).

Alkaline lead citrate (Reynolds. 1963) was prepared by mixing:

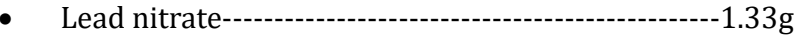

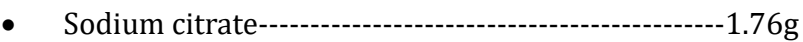

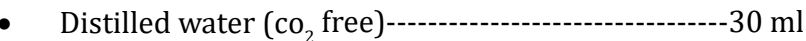

The mixture was shaken vigorously at intervals for $30 \mathrm{~min}$. in $50 \mathrm{ml}$ volumetric flask. The completion of the conversion of lead nitrate to lead citrate was indicated by the appearance of a uniform milky suspension. To this suspension was added $8 \mathrm{ml}$ of $1 \mathrm{~N} \mathrm{NaOH}$ $(4 \%)$.

It was distilled water to $50 \mathrm{ml}$ and then mixed by inversion until lead citrate dissolved and the suspension cleared up completely distilled water used for preparing lead citrate solution should be free of carbon dioxide which could be removed by boiling the water for about $8 \mathrm{~min}$. the solution was then covered and allowed to cool before use. This solution could be kept for several weeks if kept tightly closed. if precipitates appear the solution should be discarded.

\section{Staining procedure}

Separate drops of uranyl acetate were put on a parafilm in a petri dish. The drops would round up because of the hydrophobic nature of the surface of the parafilm. Each grid was put over one drop with the sections facing downwards and left for $20 \mathrm{~min}$ at room temperature with the area covered. Each grid was rinsed in three changes of $50 \%$ methanol while being held with fine-pointed forceps.

Counterstaining with lead citrate solution was carried out as with uranyl acetate for $20 \mathrm{~min}$. at room temperature protected from air by another petri dish cover. 
Pellets of sodium hydroxide were usually put beside the grids to absorb carbon dioxide during lead citrate staining to prevent the formation of lead carbonate which forms precipitate on the sections. The grids were then washed, dried, and Kept in a grid box till viewing under the EM.

\section{Electron microscopic examination}

It was performed using a Zeiss EM transmission electron microscope at $60 \mathrm{~K} \mathrm{~V}$. The grids were inserted in the specimen holder. At first low magnification was used (X 1000) to locate the specimen, then higher magnification (up to X14000) was used to identify the cells and evaluate the findings.

\section{Quantitative morphometric measurements}

The data were obtained using (Leica Qwin-C 500) image analyzer computer system (England). The image analyzer consisted of a colored video camera, colored monitor, hard dies of IBM personal computer connected to the microscope, and controlled by (Leica Qwin- C500) software. The image analyzer was first calibrated automatically to convert the measurement units (pixels) produced by the image analyzer program into actual micrometer units.

The following parameters were measured in the chosen slides

- Trabecular bone area: Defined as the percentage of Trabecular bone tissue, by measuring the area percent relative to the standard measuring frame $\left(127504.6 \mathrm{um}^{2}\right)$ using an objective lens of magnification ten (I.e. the total magnification used was 100) detected in each field. The number of fields examined was ten fields in each specimen.

- Cortical bone thickness: Defined as the combined thickness of the medial and lateral sides of cortical bone for each sample by measuring ten different fields in the midshaft of the tibia for each side and then taking the average of the two sides, using an objective lens of magnification four (i.e. the total magnification used was 40). Ten readings were recorded in each specimen.

- Degree of calcification and bone uncalcification of bone: BY measuring the area percent relative to the standard measuring frame (127504 $\left.\mathrm{um}^{2}\right)$ using an objective lens of magnification ten (i.e. the total magnification used was 100) detected in each field. It was examined in slides of undecalcified bone specimens stained with modified Masson (Gldner's) trichrome. The number of fields examined was ten fields in each specimen.
- Osteoclast area and number: By estimating the cell area and number per high power field in relation to the nearby bone trabeculae in micrometers using an objective lens of magnification 40 (i.e. the total magnification used was 400). It was most prominent in sections stained with Hx\&E. The number of fields examined was ten fields in each specimen.

- Thickness of endometrial lining epithelium: Measured from the apical surface of the epithelial columnar cells perpendicular to the basal lamina, using an objective lens of magnification ten (i.e. the total magnification used was 100). Ten readings were recorded in each specimen.

The parameters measured were the area, area percent, and the standard measuring frame detected in each field.

They were defined as:

- Area: The total area of the detected object within the measuring frame.

- $\quad$ Area percent: This is the proportion of detected pixels in the image. Expressed as a percentage from 0 to 100.

- Standard measuring frame: It is a fixed "guard region" within the image window. It is the area of measure frame (it was $127504.6 \mathrm{um}^{2}$ ) using magnification X 100.

\section{Conclusion}

The current investigation revealed that the production of diabetes was associated with significant bone loss or osteopenia, which may directly lead to persistent hyperglycemia, hormonal imbalance, and a decrease in IGF-1, with a resulting rise in bone oxidative stress. In contrast, ZSC treatment appeared to have the potential to be engaged in the prevention of diabetes-induced bone loss, mostly through the normalization of hyperglycemia and the hormonal pattern as early events, followed by the increase in IGF-1 bioavailability.

\section{Bibliography}

1. Aarts MM., et al. "Parathyroid hormone-related protein promotes quiescence and survival of serum-deprived chondrocytes by inhibiting rRNA synthesis". Journal of Biological Chemistry 276 (2001): 37934-37943.

2. Aarts MM., et al. "The nucleolar targeting signal (NTS) of parathyroid hormone-related protein mediates endocytosis and nucleolar translocation". Journal of Bone and Mineral Research 14 (1999): 1493-14503. 
3. Andreasen CH., et al. "Low physical activity accentuates the effect of the FTO rs9939609 polymorphism on body fat accumulation". Diabetes 57 (2008): 95-101.

4. Aaron JE., et al. "The microanatomy of trabecular bone loss in normal and aging men and women". Clinical Orthopaedics 215 (1987): 260-271.

5. Aerssens J., et al. "Mechanical properties, bone mineral content, and bone composition (collagen, osteocalcin, IGF1) of rat femur: Influence of ovariectomy and nandrolone decanoate (anabolic steroid) treatment". Calcified Tissue International 53 (1993): 269-277.

6. Agarwal SK and Judd HL. "Management of menopause". In: Riggs BL and Melton LJ III (eds) Osteoporosis: Etiology, diagnosis, and management. Second edition. Lippincott-Raven Publishers, Philadelphia, (1995): 351-370.

7. Aarts MM., et al. "Parathyroid hormone-related protein promotes quiescence and survival of serum-deprived chondrocytes by inhibiting rRNA synthesis". Journal of Biological Chemistry 276 (2001): 37934-37943.

8. Aarts MM., et al. "The nucleolar targewting signal (NTS) of parathyroid hormone-related protein mediates endocytosis and nucleolar translocation". Journal of Bone and Mineral Research 14 (1999): 1493-14503.

9. Adams C., et al. "Matrix regulation of skeletal cell apoptosis: Role of calcium and phosphate atoms". Journal of Biological Chemistry 276 (2001): 20316-20322.

10. Afzal F., et al. "Osteoblast growth and differentiation is retarded in endothelial nitric oxide synthase knockout mice (abstract)". Journal of Bone and Mineral Research 15 (2000): 217.

11. Adams JM. "Ways of dying: Multiple pathways to apoptosis". Genes and Development. 17 (2003): 2481-2495.

12. Aguirre J., et al. "Endothelial nitric oxide synthase gene deficient mice demonstrate marked retardation in postnatal bone formation, reduced bone volume, and defects in osteoblast maturation and activity". The American Journal of Pathology 158 (2001): 247-257.

13. Aguirre JL., et al. "The anti-apoptotic effects of mechanical stimulation in osteoblasts/osteocytes are transduced by the estrogen receptor (ER): A novel ligand-independent function of the ER". Journal of Bone and Mineral Research 18 (2003): S71.

14. Akhter MP., et al. "Effect of parathyroid hormone (HPTH [184]) treatment on bone mass and strength in ovariectomized rats". Journal of Clinical Densitometry 4 (2001): 13-23.
15. Alieva IB., et al. "Experimental model for studying the primary cilia in tissue culture cells". Membrane and Cell Biology 12 (1999): 895-905.

16. Naim HY., et al. "Biosynthesis and maturation of lactase-phlorizin hydrolase in the human small intestinal epithelial cells". Biochemical Journal 241 (1987): 427-434.

17. Boll W., et al. "Structure of the chromosomal gene and cDNAs coding for lactase-phlorizin hydrolase in humans with adulttype hyperlactation or persistence of lactase". American Journal of Human Genetics 48 (1991): 889-902.

18. Fang R., et al. "The homeodomain protein $\mathrm{Cdx} 2$ regulates lactase gene promoter activity during enterocyte differentiation". Gastroenterology 118 (2000): 115-127.

19. Mitchelmore C., et al. "Interaction between the homeodomain proteins Cdx2 and HNF1 alpha mediates expression of the lactase phlorizin hydrolase gene". Biochemical Journal 346.2 (2000): 529-535.

20. Van Wering HM., et al. "Novel interaction at the $\mathrm{Cdx}-2$ binding sites of the lactase-phlorizin hydrolase promoter". Biochemical and Biophysical Research Communications 299 (2002): 587-593.

21. Troelsen JT., et al. "Regulation of lactase-phlorizin hydrolase gene expression by the caudal-related homoeodomain protein Cdx-2". Biochemical Journal 322.3 (1997): 833-838.

22. Troelsen JT., et al. "A novel intestinal trans-factor (NF-LPH1) interacts with the lactase-phlorizin hydrolase promoter and co-varies with the enzymatic activity". Journal of Biological Chemistry 267 (1992): 20407-20411.

23. "How Many Bones Does a Baby Have and Why Do Adults Have Fewer?". Healthline (2021).

24. Steele D Gentry and Claud A Bramblett. "The Anatomy and Biology of the Human Skeleton". Texas A and M University Press (1988): 4 .

25. "Mammal anatomy: an illustrated guide". New York: Marshall Cavendish. (2010): 129.

26. “Ossein". The Free Dictionary (2020).

27. Hall John. "Textbook of Medical Physiology (12 ${ }^{\text {th }}$ edition.)". Philadelphia: Elsevier (2011): 957-960.

28. Schmidt-Nielsen Knut. "Scaling: Why Is Animal Size So Important?". Cambridge: Cambridge University Press (1984): 6.

29. Alam MW., et al. "First report of Alternaria alternata causing postharvest fruit rot of jujube in Pakistan". Plant Disease 102.2 (2018): 452-453. 
30. Morton J. "Indian Jujube". In: Fruits of warm climates. Julia F. Morton, Miami, FL. Department of Horticulture and Landscape Architecture at Purdue University (1987): 272-275.

31. Robert Bringhurst. "The Elements of Typographic Style (version 3.1)". Point Roberts, WA: Hartley and Marks (2005): 6869.

32. "Chapter 15: Footnotes, indexes, contents, and outlines". U.S. Government Printing Office Style Manual (2010).

33. Lampe Peter. "Early Christians in the City of Rome in Christians as a Religious Minority in a Multicultural City". The Library of New Testament Studies London: T and T Clark International 243 (2004): 24.

34. Janick Jules and Paull Robert E. "The encyclopedia of fruit and nuts". Centre for Agriculture and Bioscience International publishing (2008): 615-619

35. Morton J. "Indian Jujube”. In: Fruits of warm climates. Julia F. Morton, Miami, FL. Department of Horticulture and Landscape Architecture at Purdue University (1987): 272-275.

36. "Ziziphus mauritiana - a valuable tree for arid and semi-arid lands" (1998).

Volume 4 Issue 12 December 2021

(C) All rights are reserved by Omar Abdulrhman

Alfaroq., et al. 